\title{
INTERKULTURELLE KOMPETENZTRAININGS FÜR EHRENAMTLICHE IN DER FLÜCHTLINGSARBEIT AUF DEM PRÜFSTAND. STUDIE ZU DEN KONZEPTIONELLEN GRUNDLAGEN UND ZUM CRASHKURSTREND IN DER FREIWILLIGENQUALIFIZIERUNG
}

\author{
CORNELIA SPRINGER (HAMBURG)
}

\section{ABSTRACT INTERCULTURAL SKILLS TRAINING FOR VOLUNTEERS IN REFUGEE WORK PUT TO THE TEST. STUDY ON THE CONCEPTUAL FOUNDATIONS AND CRASH COURSE TREND IN VOLUNTEER QUALIFICATION}

The themes of flight and migration determine the public discourse more than ever before. Not only practical assistance is required, just as evident is the challenge for each individual in the personal interaction. Every encounter takes place in a cultural overlapping situation and is thus extraordinary. The new presence of the theme demands everyone's cultural self-location and reflection on categories of strangeness and familiarity. Intercultural competence gains a new status and a special marketability in the whole range: own and foreign cultural knowledge, self-reflection ability, change of perspective, knowledge of foreign languages. This is accompanied by a change in perception and an appreciation of the preconditions of cultural hybrids. The article examines to which theoretical concepts of interculturality refer the measures of renowned, state-subsidized institutions. The used concept of culture plays a role here, questions of quality assurance and effectiveness as well as the adequacy of theory and methods for the target group. In order to cope with the complexity of super-diverse societies, an interdisciplinary research perspective is needed. Therefore, both sources of interculturalism and cultural studies as well as sociology and political science are included.

Key words: intercultural skills, refugee work, volunteers training

\section{ABSTRAKT \\ MEZIKULTURNÍ KOMPETENCE DOBROVOLNÍKŮ PRACUJÍCÍCH S UPRCHLÍKY. STUDIE O KONCEPTUÁLNÍCH ZÁKLADECH A TRENDU K RYCHLOKURZŮM V DOBROVOLNICKÉ KVALIFIKACI}

Témata uprchlictví a migrace určují veřejný diskurz více než kdy předtím. Nejde přitom jen o praktická opatření, nýbrž zároveň o výzvu pro každého jednotlivce v osobní interakci. Každé setkání se v této oblasti uskutečňu- 
je v průniku kultur a je mimořádné. Nová aktuálnost tématu vyžaduje od každého člověka kulturní lokalizaci sebe sama i reflexi kategorií cizího a vlastního. Mezikulturní kompetence získávají nový význam a uplatnění na trhu v plné šíri jejich aspektů: znalosti vlastní a cizí kultury, schopnost sebereflexe a změny perspektiv, znalost cizích jazyků. $S$ tím je spojena změna vnímání a hodnocení předpokladů kulturně hybridních fenoménů. Tento prŕspěvek zkoumá teoretické koncepty interkulturality, s nimiž pracují renomované, státem dotované instituce v oblasti pomoci uprchlíkům. Roli zde hraje uplatňovaný pojem kultury, otázky zajištění kvality a účinnosti, jakož i přiměřenost teorie a metod pro cílovou skupinu. Vzhledem ke složitosti kulturně vysoce rozmanitých společností je zapotřebí interdisciplinární perspektivy výzkumu. Zahrnuty jsou proto jak př́stupy mezikulturních studií a vědy o kultuře, tak i sociologie a politické vědy.

Klíčová slova: mezikulturní kompetence, práce s uprchlíky, vzdělávání dobrovolníků

Die Themen Flucht und Migration bestimmen derzeit stärker denn je den öffentlichen und politischen Diskurs in Deutschland und Europa. Die Herausforderungen, die die große Zahl der Schutzsuchenden im Jahr 2015 mit sich brachte, wurden als „europäische Krise" (Daldrup 2016) beschrieben und von vielen als Gefährdung einer bestehenden wirtschaftlichen und politischen Stabilität empfunden. In der kommunalen Flüchtlingspolitik standen zunächst die Erstversorgung und Unterbringung der Ankommenden sowie die Klärung der Aufenthaltsstatus im Vordergrund, gefolgt von sprachlicher und beruflicher Integration. Staatliche Institutionen waren mit ihren umfänglichen administrativen Aufgaben überfordert. Umso wichtiger war die Rolle freiwilliger Unterstützer/-innen, die sich im ganzen Bundesgebiet an Bahnhöfen, in Kleiderkammern, Erstaufnahmeeinrichtungen u.v.m. engagierten. Ad hoc hat die Zivilgesellschaft große Verantwortung übernommen, aus dem Nichts unterstützende Strukturen geschaffen und viele Angebote, in Kooperation mit staatlichen und kirchlichen Einrichtungen, über einen langen Zeitraum aufrechterhalten.

\section{Hintergrund und Motivation der Studie: Zur Relevanz interkultureller Kompetenz für Ehrenamtliche in der Flüchtlingsarbeit}

Neben organisatorischen und praktischen Aufgaben stehen die Engagierten selbsterklärend einer Vielzahl kommunikativer Herausforderungen gegenüber, die sich in der Interaktion mit geflüchteten Menschen ergeben. Jede Begegnung findet in einer kulturellen Überschneidungssituation statt und ist somit außergewöhnlich. Wenngleich bei der deutschen Gesellschaft von einer seit vielen Generationen durch Zuwanderung geprägten ausgegangen werden muss und darf ${ }^{1}$ und interkulturelle Kommunikation ins-

1 Zur Geschichte und Verfasstheit der deutschen als Migrationsgesellschaft vgl. Broden/Mecheril (2007). 
besondere in urbanen Räumen häufig der Normalfall ist, kamen durch ihr Engagement dennoch viele ,Deutsche ${ }^{c_{2}}$ mit Menschen in Kontakt, die sich aufgrund ihrer Herkunft (Syrien, Eritrea, Afghanistan u.a.) von ihrem gewohnten sozialen Umfeld unterschieden ${ }^{3}$. In Verwaltungs- und Behördenkommunikation begrenzt der thematische und institutionelle Rahmen die Auswirkungen des differenzbedingten Potenzials für interkulturelle Missverständnisse und Konflikte. ${ }^{4}$ Soll allerdings an die Stelle einer per se temporären Willkommenskultur ${ }^{5}$ eine gemeinsam gestaltete Gesellschaft der Vielfalt treten, erhöhen sich auch Komplexität und Anforderungen interkultureller Kompetenzbereiche und ihrer Vermittlung. Auch heute kommen mit den Migrantinnen und Migranten vor allem Menschen nach Europa (vgl. Frisch 1967: 100), die Zuflucht und oftmals einen Neuanfang, nicht jedoch hiesige Fachkräfteengpässe (vgl. Vollmer 2015) auszugleichen suchen. Das Deutschland des noch jungen 21. Jahrhunderts möchte dem Narrativ der Gastarbeitergeneration ${ }^{6}$ mehrheitlich etwas optimistisch Zukunftsweisendes entgegensetzen. Die Obligation der eigenen kulturellen Verortung und der bewussten Auseinandersetzung mit Kategorien von Fremdheit und Vertrautheit ergibt sich plötzlich für jeden und jede. ,Interkulturelle Kompetenz' erlangt in professionellen wie nicht professionellen Kontexten einen neuen Stellenwert (vgl. Bruchhagen 2016) und wird in der Arbeit mit Migrant/-innen als schlicht notwendige Qualifikation vorausgesetzt. ${ }^{7}$ In erstaunlich kurzer Zeit wurde, einhergehend mit dem überwältigenden Freiwilligenengagement für Geflüchtete, ein beachtliches Angebot an kostenlosen Fortbildungen für ,Helfer/-innen entwickelt, in dem das Training interkultureller Kompetenz von Anfang an eine zentrale Rolle einnahm. ${ }^{8}$ Diese interkulturellen Fortbildungen näher zu untersuchen, war Anlass einer qualitativen Studie, die im Folgenden vorgestellt wird.

2 Zum einen ist ein Großteil der ,Flüchtlingshelfer/-innen über 50 Jahre alt und gehört einer Generation an, die im Vergleich zur jüngeren Generation, die bereits in der Schule mit einer stärkeren kulturellen Heterogenität aufwächst (vgl. Schründer-Lenzen 2009: 121), pauschal weniger direkte Erfahrung mit sogenannten ,Menschen mit Migrationshintergrund' hat. 2015 hat die Anzahl der Älteren im Vergleich zu 2014 zudem stark zu-, die der unter 30-Jährigen stark abgenommen (vgl. Karakayali/Kleist 2016: 13). Zum anderen ist in ländlicheren Regionen der Kontakt zu Menschen mit Migrationserfahrung weniger ,normal' als in städtischen Räumen. Das Engagement in kleineren Gemeinden war jedoch groß, sodass „der Anteil der Ehrenamtlichen an Landstädten [...] sich von knapp 4 auf 16 Prozent vervierfacht [hat und] der Anteil der Engagierten in den Kleinstädten von 11,1 auf 19 Prozent angewachsen [ist]“ (Ebd.: 16).

3 Zu den Hauptherkunftsländern vgl. die Asylgeschäftsstatistik des BAMF: Bundesamt für Migration und Flüchtlinge (2016b: 2). Es kann in der Arbeit mit Geflüchteten eine mehrdimensionale Befremdung postuliert werden, bedingt durch Differenzen in Sprache, Religion und Weltanschauung, politischer Systeme u.a.

$4 \mathrm{Zu}$ den Besonderheiten interkultureller Behördenkommunikation liefert Rosenberg (2014) eine differenzierte gesprächsanalytische Untersuchung.

5 Denn im Willkommen steckt eine Begrüßung, ein Empfangen, und dieses bezieht sich sinnvoll nur auf eine Phase des Anfangs und Übergangs, bis zu einem perspektivischen Ankommen und Dazugehören der ,Neuen' in der Aufnahmegesellschaft.

6 In den 60er und 70er Jahren Zugewanderte fühlen sich häufig selbst nach Jahrzehnten nicht in der deutschen Gesellschaft angekommen und angenommen (vgl. Geißler 2014).

7 Viele große Themen werden mit interkultureller Kompetenz assoziiert: Kultur und Kommunikation, persönliche Haltung und Einstellung, Werte wie Nächstenliebe, Menschlichkeit und Gerechtigkeit. Eine Definition liefert z.B. Bolten 2007. In den hier untersuchten Veranstaltungen wurde der Begriff selbst nicht problematisiert, sondern lag die Konzentration eher auf der Klärung des Kulturbegriffs.

8 Natürlich werden im Zuge der Freiwilligenqualifizierung diverse Themen geschult. Neben einer Vermittlung von, man könnte sie so bezeichnen, ,Hard Skills in der Flüchtlingsarbeit' (Grundlagen im Asyl- und Ausländerrecht, Familienrecht, Arbeitsrecht usw., Grundlagenwissen Islam, Kultur und 


\section{Studie zu interkulturellen Kompetenztrainings für Ehrenamtliche in der Flüchtlingsarbeit. Vorgehen und Methode}

Gegenstand der Studie waren ausschließlich interkulturelle Fortbildungsangebote staatlich oder kirchlich subventionierter Träger, die exklusiv für Ehrenamtliche (d.h. in vielen Fällen für Laien und Unprofessionelle) in der Flüchtlingsarbeit konzipiert und kostenlos angeboten wurden. Es sollte untersucht werden, ob diese eigens geschaffenen Maßnahmen der besonderen Zielgruppe gerecht werden und einen Beitrag zu ihrer Unterstützung in der Praxis leisten können, oder signifikante Defizite vorliegen, für deren Behebung sich Handlungsempfehlungen aussprechen ließen. Auf Basis einer Recherche und Systematisierung von Fortbildungen, die auf einschlägigen Portalen oder Trägerseiten im Internet, in elektronischen Rundbriefen und Flyern beworben wurden, wurden drei Hypothesen formuliert (s. Abschnitt 4) und in der anschließenden Feldphase überprüft. ${ }^{9}$ Es wurden verschiedene Feldzugänge gewählt. Nicht zuletzt mit Blick auf das Vorhaben, in einer Auswahl von Veranstaltungen zu hospitieren, lag der Schwerpunkt dabei auf Hamburg. Hier bestand und besteht ein, auch verglichen mit anderen Kommunen, sehr breites Fortbildungsangebot. Daneben wurden, per Stichwortsuche ${ }^{10}$, Veranstaltungen in anderen deutschen Städten gesichtet und in einigen Fällen persönlich Kontakt für ein Interview aufgenommen.

Mit Vertreter/-innen von Trägern, die Fortbildungen für Freiwillige in der Flüchtlingsarbeit koordinieren und fördern, und von Einrichtungen, die solche durchführen, sowie mit Trainerinnen, die Fortbildungen konzipieren und leiten, wurde eine Reihe leitfadenbasierter Telefoninterviews geführt (vgl. Tab. 3 und Tab. 4). ${ }^{11}$ Von sämtlichen Interviews liegen kommentierte Protokolle vor, die in die folgenden Ausführungen einfließen. Eine Tonaufzeichnung war in keinem Fall erwünscht. Teilnehmer/-innen von Fortbildungen wurden bislang nicht befragt, allerdings konnten in der Studie Äußerungen und Positionen von Teilnehmer/-innen einbezogen werden, die durch Protokolle während der Teilnahme und Hospitation in einer Auswahl von Veranstaltungen dokumentiert wurden. ${ }^{12}$ Anhand eines Analyserasters (vgl. Tab. 2) wurden u.a. die von Veranstaltern

Politik der Herkunftsregionen, Fluchtursachen, Fluchtrouten und Fluchtpunkte u.a.) stehen, Soft und Social Skills in der Flüchtlingsarbeit' hoch im Kurs(programm), darunter: Gelingendes Miteinander im Team mit anderen Ehrenamtlichen, Freiwilligenkoordination, Zeit- und Projektmanagement, Selbstreflexion/ Rollen und Grenzen des Engagements, traumasensibler Umgang mit Geflüchteten (vgl. z.B. auch Stiftung Bürgermut/Bundesarbeitsgemeinschaft der Freiwilligenagenturen e.V. 2016: $101)$.

9 Eine ausführliche Analyse der Ankündigungstexte hinsichtlich des ,Wordings, formulierter Vermittlungs- bzw. Lernziele etc., würde hier den Rahmen sprengen. In der Übersicht im Anhang (Tab. 1) werden daher nur die Titel der Fortbildungsveranstaltungen aufgeführt. Feststellbar ist, dass die Bezeichnungen seit Beginn der Etablierung umfangreicher Fortbildungsangebote für diese Zielgruppe zunehmend unterschiedlicher und differenzierter werden. Das ist zum einen Ausdruck einer wachsenden Sensibilität und Erfahrung bei den Trägern und eines präziseren Zielgruppendesigns, zum anderen einer inzwischen initiierten inhaltlichen Ausdifferenzierung und Weiterentwicklung der Angebote, die zum Zeitpunkt der Erhebung noch nicht in diesem Maße vorlag.

10 Stichwörter: interkulturelle Kompetenz, interkulturelle Sensibilisierung, Flüchtlingsarbeit, Flüchtlingshilfe, Ehrenamt, Qualifizierung, Fortbildung u.a.

11 In ausgenommenen Fällen wurden Trainerinnen in Präsenz befragt.

12 Mit der durchgeführten Erhebung und den daraus gewonnenen Erkenntnissen wird ausdrücklich kein Anspruch auf Repräsentativität erhoben. Die Bildung der Stichprobe von für das Forschungsvorhaben 
und Trägern von interkulturellen Fortbildungen formulierten Vermittlungsziele, in den Fortbildungen rekurrierte theoretische Konzepte von Interkulturalität, der zitierte Kulturbegriff und angewandte Kulturerfassungsansätze untersucht. Überdies wurden Formate und Umfang der Angebote mit Blick auf Qualitätssicherung, inhaltlich-thematische Adäquatheit sowie methodisch-didaktische Umsetzung verglichen und in ihrer Angemessenheit für die Qualifizierung von Freiwilligen in der Flüchtlingsarbeit hinterfragt. Die wichtigsten Ergebnisse sollen im Folgenden besprochen werden.

\section{Interviews und Hospitationen}

\subsection{Interviewreihe 1: Trägereinrichtungen und Veranstalter/-innen}

Die Landschaft der Träger und Anbieter von Fortbildungen entspricht in ihrer Vielfalt der von Akteur/-innen in der Flüchtlingsarbeit allgemein. Darunter sind sowohl staatliche als auch kirchliche Einrichtungen, Bürgerinitiativen, gemeinnützige Vereine u.v.m. Unter den hier einbezogenen Trägern und Anbietern waren das Diakonische Werk Hamburg, der Paritätische Wohlfahrtsverband Hamburg und der Landesbetrieb ZAF/AMD. ${ }^{13}$ Daneben wurden Mitarbeiter/-innen von Volkshochschulen, des Caritasverbands für die Stadt Köln und des Hamburger Instituts für Interkulturelle Pädagogik (HIIP) konsultiert (vgl. Tab. 4). ${ }^{14}$ Freiwilligenagenturen, Volkshochschulen und Bildungswerke von Wohlfahrtsorganisationen sind originär als Träger in der Freiwilligenqualifizierung aktiv. So können sie auf die notwendige Expertise zurückgreifen, wenn sie sich die Fortbildung Ehrenamtlicher in der Flüchtlingsarbeit als neuen Teil ihres Aktionsfelds erschließen. Als eigene Motivation gaben alle Befragten an, das Ehrenamt bzw. Freiwilligenengagement fördern und zur qualitativen Verbesserung desselben beitragen zu wollen. Die Finanzierung der hier betrachteten Maßnahmen erfolgte durch öffentliche/ staatliche, kirchliche oder von Stiftungen bereitgestellte Mittel sowie im Rahmen der Förderung von Multiplikatorenschulungen durch das Bundesamt für Migration und Flüchtlinge (BAMF) (vgl. Bundeszentrale für politische Bildung 2016: 1). Um der Vergleichbarkeit willen wurden nur Fortbildungen einbezogen, die die Ehrenamtlichen ohne Teilnahmegebühr wahrnehmen konnten.

relevanten Einrichtungen und Personen erfolgte nach theoretischen und pragmatischen Kriterien, etwa dem leichteren persönlichen Zugang zu Veranstaltungen in Hamburg. Es sei aber darauf hingewiesen, dass die Studie sich, indem sie den Fokus auf interkulturelle Sensibilisierungsmaßnahmen für Ehrenamtliche in der Flüchtlingsarbeit richtet, einem bislang nicht/ kaum erforschten Feld der Freiwilligenqualifizierung widmet und somit viele Anschlussmöglichkeiten für die weitere Forschungsarbeit aufzeigt.

13 Der Landesbetrieb ZAF/AMD ist zentraler Dienstleister in der Aus- und Fortbildung sowie Arbeitsmedizin für die hamburgische Verwaltung. Fortbildungen für Ehrenamtliche in der Flüchtlingsarbeit gehören zum Unterstützungsangebot des Forums Flüchtlingshilfe der Behörde für Arbeit, Soziales, Familie und Integration (BASFI).

14 Weitere Träger und Veranstalter gehen aus Tab. 1 hervor. 
Weiter wurden Trainerinnen von interkulturellen Fortbildungen interviewt (vgl. Tab. 4). Ausnahmslos alle Befragten sind weiblich und verfügen über eine professionelle (Zusatz-)Ausbildung zur interkulturellen Trainerin. Die meisten sind zudem als Systemische Coaches, Mediatorinnen oder (Organisations-)Beraterinnen ausgebildet und haben langjährige Erfahrung in der Erwachsenenbildung und Moderation von Trainings. Einige hatten in anderen Themenfeldern bereits viel Expertise in der Qualifizierung von Ehrenamtlichen, andere waren darin zum Zeitpunkt der Befragung noch ganz neu. Einige hatten noch wenig bis gar keine Erfahrung mit einer Klientel, die in der Flüchtlingsarbeit engagiert ist, sondern primär mit Teilnehmer/-innen aus international arbeitenden Unternehmen, öffentlicher Verwaltung und Kirche. Die Trainerinnen waren nahezu alle externe Auftragnehmerinnen, die nicht fest zum Team der Trägereinrichtungen gehörten. So setzten letztere ausgewiesene Fachkräfte ein, denen sie in der Gestaltung der Schulungen, wie aus allen Interviews hervorging, völlige Freiheit ließen. Dies war einerseits Ausdruck von Vertrauen in die Kompetenz der Expert/-innen. In den Interviews konnten die befragten Auftraggeber/-innen allerdings leider kaum eine Aussage über Inhalte und Methoden der Fortbildungen treffen. ${ }^{15}$

\subsection{Hospitation und Teilnahme an Fortbildungsveranstaltungen}

Bei Hospitationen und der aktiven Teilnahme an Fortbildungen (vgl. Tab. 5) wurden der Schulungsverlauf und relevante mündliche Äußerungen von Trainerinnen und Teilnehmer/-innen protokolliert. Tonaufzeichnungen waren auch hier nicht erwünscht und hätten vermutlich zudem starken Einfluss auf die Gruppe genommen. Die Teilnahme an einigen Veranstaltungen war aktiv und partizipativ angelegt, bei einigen wurde nur teilnehmende Beobachtung angestrebt, um eine distanziertere Perspektive zu gewährleisten.

\section{Interkulturelle Sensibilisierungsmaßnahmen für Ehrenamtliche in der Flüchtlingsarbeit auf dem Prüfstand}

Im Vorfeld der Interviews und Hospitationen wurden drei Hypothesen aufgestellt, um die Konzeption, Durchführung und Wirksamkeit interkultureller Fortbildungen für Freiwillige in der Flüchtlingsarbeit zu untersuchen. Im Folgenden werden diese diskutiert und versucht Handlungsempfehlungen für die Praxis der Freiwilligenqualifizierung abzuleiten.

15 Inzwischen werden in einigen der konsultierten Einrichtungen Fortbildungen zur interkulturellen Kompetenzentwicklung auch von eigenen Mitarbeiter/-innen angeboten, die sich zu Trainer/-innen weiterqualifiziert haben. 


\subsection{Zielgruppenspezifische Angebote}

Hypothese 1: ,Die Gruppe der Teilnehmer/-innen ist in ihrer Zusammensetzung stark heterogen, überdurchschnittlich motiviert, hat ein knappes Zeitbudget und einen hohen Qualitätsanspruch an Fortbildungen. Sie nimmt diese nur dann wahr, wenn sie ihrer Erwartungshaltung entsprechen und praxisrelevant sind. Daher ist das Zielgruppendesign für die Adäquatheit der Maßnahmen ausschlaggebend.'

Durch die Interviews und teilnehmende Beobachtung wurde die Annahme der stark heterogenen Gruppenzusammensetzung bestätigt. Sie spiegelt die in statistischen Erhebungen festgestellte Struktur der Freiwilligen in der Flüchtlingsarbeit wider, die z.B. die EFA-Studie (Karakayali/Kleist 2016: 11ff.) oder der Freiwilligensurvey (Simonson/Vogel/ Tesch-Römer 2014: 95ff.) beschreiben. Die Gruppe der ,Helfer/-innen' charakterisiert eine hohe Diversität der Lernvoraussetzungen, darunter professionelle und/oder private Vorerfahrungen mit Migration, kultureller Diversität und Flüchtlingsarbeit, ${ }^{16}$ soziokulturelle, religiöse u.a. Prägungen, persönliche Haltungen und Einstellungen, Motivationen für das Engagement ${ }^{17}$ und das Alter der Teilnehmer/-innen. Die (hohe) Erwartungshaltung von Ehrenamtlichen wird zu zwei Zeitpunkten in der Fortbildung besonders deutlich. $\mathrm{Zu}$ Beginn fragt die Trainerin meist ab, welche Lernziele und eigenen Fragen die Teilnehmer/-innen mitbringen. Das Spektrum ist umfangreich und zudem unscharf, angefangen bei rechtlichen Fragestellungen, über Unsicherheiten im Umgang mit religiösen Menschen bis hin zu weltpolitischen Themen (vgl. Tab. 6 im Anhang). Aus den Rückmeldungen wird deutlich, dass das Anliegen der Teilnehmer/-innen ein dezidiert praxisnahes und handlungsrelevantes ist, die Lernerwartungen weit über das Feld der interkulturellen Kommunikation hinausgehen und vielmehr alle Bereiche der ehrenamtlichen Flüchtlingsarbeit betreffen. ${ }^{18}$ Dieser thematischen Breite kann (und will) ein interkulturelles Training nicht gerecht werden. Entsprechend reagierten die Trainerinnen auf die formulierten Erwartungen und erläuterten, worin das Ziel der Fortbildung besteht, was sie leisten kann - und was nicht. Ferner ist erwähnenswert, dass abschließendes mündliches oder schriftliches Feedback häufig nicht nur wohlwollend ausfällt, wie es bei kostenlosen Angeboten vielleicht zu erwarten wäre, sondern mitunter durchaus kritisch.

Schon an dieser Stelle ergibt sich die Frage, ob interkulturelle Trainingsangebote der Zielgruppe überhaupt gerecht werden können. Diese Frage wird in den weiteren Ausführungen wieder aufgegriffen. Vorläufig lässt sich festhalten, dass ein Zielgruppendesign hier die Gewährleistung einer mehrdimensionalen Adäquatheit der Angebote erfordert. Wie erwähnt, müssen Inhalte und Themen, um für die Teilnehmer/-innen relevant zu sein, explizit Praxisbezüge zur Arbeit mit Geflüchteten aufzeigen. Vermittelte Modelle und Methoden sind nur dann nützlich, wenn die Freiwilligen damit später eigenständig arbeiten und sie anwenden können. Statt eines hohen Theorieanteils kommt es auf einen

$16 \mathrm{Zu}$ den von den Teilnehmer/-innen angegebenen Engagementfeldern zählen Patenschaften, Vormundschaften, Tandems, Lernbegleitung, Behördenbegleitung, Wohnungs- und Jobsuche u.a.

17 Z.B. Neugierde, der Wunsch zu helfen, eigene berufliche Weiterqualifizierung etc.

18 Daneben nimmt der Erfahrungsaustausch mit, Gleichgesinnten, die sich in ähnlichen Situationen und Kontexten bewegen, eine wichtige Rolle ein. Mehrfach beschrieben Teilnehmer/-innen die Fortbildung als geschützten Raum, in dem Fragen zulässig seien, die sonst Regeln der Political Correctness unterlägen oder gar tabuisiert wären. 
Transfer auf authentische Handlungskontexte an. Darüber hinaus stehen viele ehrenamtlich Engagierte im Berufsleben und können tagsüber keine Fortbildungsangebote wahrnehmen. Zudem sind zeitlich sehr umfangreiche Maßnahmen für diese Zielgruppe weniger attraktiv. Diesen Kriterien werden alle betrachteten Angebote gerecht. Sie sind berufsbegleitend konzipiert, finden vor allem abends oder am Wochenende statt und sind in der Mehrheit in kompaktem dreistündigem Format gehalten. Inwieweit unter diesen Bedingungen eine Sicherung von Qualität und Nachhaltigkeit gegeben ist, soll unten noch besprochen werden.

\subsection{Einsatz interkultureller Trainingsmethoden}

Hypothese 2: ,Da Kommunikation zwischen Ehrenamtlichen und Geflüchteten grundlegend anderen situativen Rahmenbedingungen unterliegt als die von Mitarbeiter/-innen internationaler Unternehmen im Auslandseinsatz, ist ein Transfer bestehender Fortbildungskonzepte und -methoden nur bedingt sinnvoll und möglich, sodass es der Modifizierung bzw. Entwicklung alternativer Konzepte bedarf.'

Interkulturelle Trainings wurden ursprünglich zur Vorbereitung „von ins Ausland zu entsendenden Mitarbeitern (z.B. Expatriate) auf andersartige Kulturräume sowie einer Optimierung des Interaktionsverhaltens zwischen Angehörigen unterschiedlicher Kulturen“ (vgl. Springer Gabler Wirtschaftslexikon) entwickelt, d.h. auf Situationen im Rahmen professioneller Kommunikation in einer fremdkulturellen Umgebung. Die Handlungs- und kommunikativen Kontexte von Ehrenamtlichen in der Flüchtlingsarbeit sind damit nur begrenzt vergleichbar. Sie sind i.d.R. nicht professionell, vor allem aber konstituiert sich der Raum der Interaktion grundlegend anders als in der Theorie, zumal sich die ,deutsche' Migrationsgesellschaft, die als postmigrantische und superdiverse beschrieben wird (Vertovec 2007), durch eine Vielfalt hybrider und pluraler Identitäten auszeichnet (Foroutan/Schäfer 2009).

Die in den Schulungen verwandten Kulturmodelle und Kulturerfassungsansätze reduzieren Komplexität, um ,Kultur' greifbar zu machen. Das Verstehen dieser Theorien und das Übertragen auf Handlungskontexte erfordert Zeit und Übung. Insbesondere im dreistündigen Kompaktkurs-Format ist ihr Einsatz kritisch zu betrachten. Häufig wird innerhalb eines Workshops ein ganzes Set von Kulturerfassungsansätzen und Modellen eingeführt. Die Teilnehmer/-innen lernen im Schnelldurchlauf die ,Klassiker ' kennen, darunter das Kulturdimensionenmodell (Hall/Hall 1990), die Kulturzwiebel (Hofstede 2001) und das Eisbergmodell von Kultur (Ruch/Zimbardo 1974: 367), Kulturstandards (Thomas 2003) und Kulturgrammatik (Hall 1992/1994), Kommunikationsquadrat und Kulturbrille (Kumbier/Schulz von Thun 2006). Die eingesetzten Modelle und Ansätze können hier aus Platzgründen nicht näher präsentiert und besprochen werden. Dennoch wird bereits aus diesem Fächer ersichtlich, dass in der Fortbildung ein Anwenden der Modelle auf konkrete kommunikative Situationen allein zeitlich nicht möglich ist. Eine explizite Benennung und Einordnung der Konzepte durch die Trainerinnen erfolgt nicht konsequent. In den Gesprächen gaben einzelne an, die Teilnehmer/-innen nicht unnötig mit Informationen überfrachten zu wollen. Allerdings wird durch die Aneinanderreihung und teils Verschränkung suggeriert, dass alle Modelle gleichermaßen passend 
seien, um Kultur(en) zu beschreiben und Verhalten von Menschen mit ,anderem' kulturellen Hintergrund zu erklären. Zudem sind sie in ihrer Einfachheit bestechend einprägsam. Dies birgt die Gefahr, dass komplexere Lernprozesse überlagert bzw. verdrängt werden. Die Teilnehmer/-innen verlassen die Schulung, wie aus einigen Äußerungen hervorging, mitunter mit einem diffusen ,Patchwork-Wissen' von Kultur und von theoretischen Regeln und Leitsätzen interkultureller Kommunikation. Zur Illustration sei ein typisches Beispiel angeführt, das in fast jeder Gruppe zur Sprache kam. Die divergierende Auffassung von ,Pünktlichkeit‘ bei Verabredungen führt regelmäßig zu Irritationen. Hier kann es hilfreich sein, sich der Dimension der Zeitorientierung (Monochronismus/Polychronismus) in Kulturen bewusst zu sein, um z.B. nicht persönlich gekränkt zu reagieren (Hall/Hall 1990). Allerdings ist damit nicht jede Verspätung zu erklären und besteht zudem die Gefahr einer Kulturalisierung von individuell, situativ, sozial, oder institutionell bedingten Handlungen. Indem die Konzentration auf dem Verstehenwollen, anderer, fremder Kulturen' und dem Wunsch liegt, ,kulturelle Differenzen' und ,interkulturelle Konflikte` zu vermeiden, gelingt u.U. die Wahrnehmung des Individuums nicht (mehr), das womöglich schlicht die Armbanduhr vergessen oder den Bus verpasst hat und deshalb nicht zur vereinbarten Zeit erscheint. Hinweise auf kulturalisierende Verallgemeinerungen geben Teilnehmeräußerungen in jeder Fortbildung, wenn konsequent von „den Arabern“, „den Afrikanern“ und „den Flüchtlingen“ gesprochen wird, die „anders ticken“ als „die Deutschen“. Selbst wenn die Trainerin nachdrücklich darauf hinweist, dass Kulturen keine einheitlichen Gebilde und Verallgemeinerungen bzw. Pauschalisierungen unreflektiert und teils sogar Ausdruck rassistischer Denkmuster sind, können internierte Wir-Die-Dichotomien nur schwer wieder aufgeweicht werden. Verstärkend wirkt zudem die meist homogene Zusammensetzung der Lernergruppen von überwiegend Menschen ohne Migrationserfahrung und die daraus resultierende Eindimensionalität des Erfahrungsschatzes und Referenzrahmens. Durch die intensive und auch persönlich enge Arbeit mit „ihren Syrern“ oder „ihren Eritreern“ machen die Freiwilligen über einen z.T. langen Zeitraum umfangreiche Erfahrungen und finden eigenständig Erklärungen für Ereignisse in Verhalten und Kommunikation, bei denen ihre vertrauten Deutungsroutinen versagen. Das wechselseitige Bestätigen der Perspektive auf ,die Flüchtlinge، in der Teilnehmergruppe und das damit einhergehende ,Othering' führen sehr wahrscheinlich noch zu einer Verfestigung von Schablonendenken. Ferner waren auch die Trainerinnen, mit nur einer Ausnahme, keine ,People of Color' (PoC). Dadurch wurde das ,Othering', ohne sozusagen relativierende Instanz, vermutlich verstärkt.

Die untersuchten Fortbildungen waren sämtlich stark beteiligungsorientiert gestaltet. Zwar erfolgte die Vermittlung von Modellen durch frontalen Input, große Anteile bestanden jedoch in interaktiven Phasen. In diesen kamen interkulturelle Trainingsmethoden wie Simulationen oder Rollenspiele zum Einsatz, Gruppenarbeit und Reflexionsrunden, Sequenzen Kollegialer Beratung u.a. ${ }^{19}$ Diese lockern die Lernsituation auf, regen den Austausch zwischen den Teilnehmer/-innen an, dienen der Verfestigung, Anwendung und Übertragung der gehörten Theorie. In den meisten Fortbildungen gelang die Applikation der Methoden auf kommunikative Situationen in der freiwilligen Flüchtlingsarbeit. Viele dieser Methoden sind allerdings sehr zeitintensiv und erfordern eine ausführliche

19 Z.B. Bafa bafa, Barnga u.a. (vgl. Bernhard 2002: 201 und IJAB: Methodenbox Interkulturell). 
Nachbesprechung und Reflexion, um bewusste Lernprozesse anzustoßen. In zeitlich eng kalkulierten Kursen ist ihr Einsatz daher umso kritischer zu betrachten. Entscheidend ist eine ausgewogene Methodenmischung, bei der sich „[k] reative und kooperative Phasen [und] theoretische[] Inputs oder stille[] Einzelarbeit" abwechseln (Hofmann/Mau-Endres/Ufholz 2005: 26). Dies war nicht immer gegeben. Teilnehmerrückmeldungen hierzu fielen unterschiedlich aus. Lerner/-innen, die noch nicht mit interkulturellen Trainingsmethoden gearbeitet hatten, reagierten $\mathrm{z}$. T. begeistert und konnten präzise individuelle Aha-Effekte benennen. Andere kommentierten „das Spielen“ als „Zeitverschwendung“ und für die Schulung von Erwachsenen nicht angemessen.

\subsection{Alternative Theorien und Trainingsansätze}

An den im Laufe der Fortbildungen geäußerten Teilnehmerfragen (vgl. Tab. 6) wird eine Vielzahl thematischer Bereiche sichtbar, die für die praktische Flüchtlingsarbeit relevant sind, von interkulturellen Kompetenztrainings aber nicht abgedeckt werden. Besonders evident wurde dies bei der Besprechung authentischer Fälle, deren Bearbeitung häufig einer Supervision oder rechtlichen Beratung bedurft hätte. Daneben aber wurden Fragen angesprochen, die den Rückgriff auf andere reflexionsorientierte Ansätze und Theorien nahelegen. In einigen Workshops wurde der beschriebene ,Standard' der theoretischen Referenzen aufgebrochen und erweitert. Drei Beispiele mit Potential für eine Weiterentwicklung sollen hier angeführt, können aber nicht ausführlicher besprochen werden.

In einer Fortbildung wurde, unter anderem, der Anti-Bias-Ansatz aus der antidiskriminierenden Bildungsarbeit vorgestellt. Er möchte „eine intensive erfahrungsorientierte Auseinandersetzung mit Macht und Diskriminierung [...] ermöglichen und die Entwicklung alternativer Handlungsansätze zu diskriminierenden Kommunikations- und Interaktionsformen [...] fördern. “20 Diese Ziele verfolgt er durch intensive Selbstreflexion, Austausch mit anderen und anschließende Entwicklung von Handlungsmöglichkeiten. Die rassismuskritische Migrationspädagogik wurde ebenfalls in einer Fortbildung erwähnt. Sie geht davon aus, „dass wir in einer Gesellschaft leben, die von sozialer Ungleichheit geprägt ist und in der Rassismus eine zentrale Funktion für die (Re-)Produktion von Ungleichheit hat“. Ihr Ziel ist es, einen „Raum für eine Auseinandersetzung mit Migration und Rassismus und der jeweils eigenen Positionierung in diesen Verhältnissen zu schaffen.“ Allerdings setzt ein „differenzierter Blick auf die Anderen [...] den Blick in den Spiegel voraus. Mehrheitsangehörige, die professionell [oder ehrenamtlich, Anm. d. A.] mit von Rassismus und Diskriminierung betroffenen Menschen arbeiten, müssen sich mit der eigenen Verstrickung in die postkoloniale, rassistische Gesellschaft auseinandersetzen. “21 In dem Workshop wurde diese Theorie durch das Modell von Foitzik (2013) ergänzt, bei dem ein „multiperspektivischer Blick“ durch die bewusste Anwendung verschiedener „Brillen“ trainiert wird, darunter die „Kulturbrille“, „Migrationsbrille“, eine „soziale Brille“ und eine „Rassismusbrille“ (Foitzik 2013).

20 Vgl. die Website der Anti-Bias-Werkstatt, online unter: http://www.anti-bias-werkstatt.de/?q=de /content/was-ist-der-anti-bias-ansatz [Stand: 02.03.2017] sowie Herdel (2007).

21 Netzwerk Rassismuskritische Migrationspädagogik BW, online unter: http://www.rassismuskritik-bw.de/ueber-uns/positionierung/ [Stand: 11.03.2017]; Vgl. auch Atali-Timmer/Mecheril 2015 und Mecheril et al 2010. 
Der Transfer der Brillenmetapher auf eigene Handlungskontexte gelang den Teilnehmer/-innen gut. Sie verspricht auch nach der Fortbildung ein hilfreiches Werkzeug zur Selbstreflexion zu sein. Weiter hat sich als ein Baustein interkultureller Sensibilisierung die Auseinandersetzung mit Critical Whiteness-/Kritischer Weißseinsforschung (Arndt 2005) und die Reflexion ,weißer Privilegien` in postkolonialen Gesellschaften bewährt. An der Universität Hamburg wurde hierzu ein ganztägiger Workshop für freiwillig in der Flüchtlingsarbeit engagierte Studierende angeboten. ${ }^{22}$ Der zeitliche Umfang erwies sich als geeignet, da auch der Critical Whiteness-Ansatz komplex und ebenso schwer in komprimierte Fortbildungen einzubinden ist wie die anderen genannten Alternativen.

Als gemeinsames bzw. übergeordnetes Ziel aller Ansätze kann das Erreichen eines bewussten, kultur- bzw. differenzsensiblen und rassismuskritischen Umgangs mit Sprache aufgefasst werden. Dessen Relevanz liegt in der Wirkmacht von Sprache begründet, die Realität schaffen kann. „Wo vor dem Hintergrund von natio-ethno-kulturellen Abstammungs- oder Herkunftskonstruktionen körperliche und soziale Ausprägungen als Hinweis auf Unterschiede kommuniziert werden und dies mit Degradierung [...] einhergeht" (Cicek/Heinemann/Mecheril 2014: 310), liegen rassistische Denkmuster vor. In Teilnehmeräußerungen drückte sich aus, dass ein bewusster Sprachgebrauch trotz großer Empathiefähigkeit und Aufgeschlossenheit der Engagierten für Menschen mit Fluchterfahrung nicht vorausgesetzt werden kann. Denkbar wäre hier die Entwicklung von linguistisch basierten Fortbildungsmaßnahmen, ggf. in Anlehnung an das Konzept der „Linguistic Awareness of Cultures“ (LAC) von Müller-Jacquier (Vgl. Müller-Jacquier 2000 und Bosse 2009).

Die hier angedeutete Integration von alternativen Ansätzen in der interkulturellen Fortbildung von Ehrenamtlichen ist vielversprechend, weil es auf diesem Weg möglich scheint, den starken Fokus auf ,Kultur' (und damit eine nicht intendierte Kulturalisierung) zu umgehen und darüber hinaus an weitere für die Flüchtlingsarbeit relevante Themenfelder anzuschließen. Dennoch ist unabhängig von der zugrunde gelegten Theorie die Wahl der Formate ausschlaggebend. Auf diese bezieht sich die dritte Hypothese.

\subsection{Crashkurs-Formate unter kritischer Perspektive}

Hypothese 3: ,Interkultureller Kompetenzerwerb ist ein komplexer und langwieriger Prozess. Im Widerspruch dazu stehen Crashkurs-Verfahren in Fortbildungsmaßnahmen. Bei der (Weiter-)Entwicklung entsprechender Angebote sollten Wirksamkeit der Formate und Nachhaltigkeit im Fokus stehen.'

Vor allem erfahrungsorientierte Methoden entfalten ihre Wirkung durch Übung und (im Idealfall mehrstufige) Reflexion. Nach einer einmaligen Erwähnung oder Vorstellung z.B. rassismuskritischer Theorie in einem dreistündigen Kompaktkurs, der zudem in den Abendstunden stattfindet, ist evident nicht mit dem Ergebnis einer, Sensibilisierung 'bzw. ,Verinnerlichung' zu rechnen. Die Entwicklung interkultureller Handlungskompetenzen

22 Workshop im Rahmen des Qualifizierungsprogramms „Refugees welcome - aber wie?“: „Critical Whiteness - Weißsein erleben. kritische Begegnung mit einem Privileg", unter Leitung des Critical Whiteness- und Practical Black Consciousness-Trainers Tsepo Bollwinkel (vgl. http://refugees -welcome.blogs.uni-hamburg.de/16-januar-2017/ [Stand: 06.12.2017]. 
ist ein langwieriger und komplexer Prozess, der letztlich nie abgeschlossen ist und vom Einzelnen die Bereitschaft erfordert, die persönliche Haltung zu reflektieren und sich auf ein lebenslanges Lernen einzulassen. ${ }^{23}$ Mindestens sollte daher die Möglichkeit bestehen, das Gelernte nach einigen Wochen oder Monaten noch einmal aufzufrischen und in einen Erfahrungsaustausch mit Kolleg/-innen zu gehen.

Viele Träger und Veranstalter beschreiben die Kurse für Ehrenamtliche als eine (verglichen mit interkulturellen Schulungen für Professionelle) hinsichtlich Zeitumfang und ,theoretischer Aufladung' abgespeckte, d.h. niedrigschwellige Version von Trainings, sozusagen als ,interkulturelle Trainings light'. Ehrenamtliche nehmen Fortbildungen freiwillig und in ihrer Freizeit wahr. Daher entsprechen ,wenig aufwändige‘ Maßnahmen theoretisch eher ihrem Zeitbudget als Ganztagesworkshops oder mehrteilige Fortbildungen. Es muss aber hinterfragt werden, ob diese Angebote überhaupt den Lernerfolg und die Qualität erbringen können, die dem wichtigen Tätigkeitsfeld der Zielgruppe sowie ihrem eigenen Anspruch an ihr Engagement gerecht werden. Eine methodisch-didaktische Anpassung an die Bedürfnisse der Adressatengruppe, darunter stärkere Erfahrungs- und Praxisorientierung, mehr interaktive und spielerische Elemente, dafür weniger Theorie als bei einer professionellen Zielgruppe, muss nicht mit extremer zeitlicher Komprimierung einhergehen. Nicht die Relevanz interkultureller Sensibilisierung ist in Frage zu stellen - aber die gegebenen Formate. Ein Teilnehmer äußerte in der Schlussrunde: „Was ich mitnehme: an dem Thema muss ich dranbleiben. Es reicht nicht, wenn man sich das einmal anhört. Man muss das in die Praxis bringen und an sich selbst arbeiten."

Viele Teilnehmer/-innen in den in dieser Untersuchung berücksichtigten Fortbildungen hatten bereits zuvor an ähnlichen Veranstaltungen teilgenommen und für sich erkannt, dass sie in diesem Bereich mehr lernen möchten. Eine Vertiefung und Weiterarbeit mit dem Gelernten ist aber nicht möglich, wenn alle Angebote sich gewissermaßen an ,Null-Anfänger' richten und über das Kennenlernen von Modellen und das Anwenden anhand spielerischer und interaktiver Elemente nicht hinausgehen (können). Auch die Mehrheit der Trainerinnen artikulierte in den Interviews ihre Unzufriedenheit mit den Rahmenbedingungen - wenngleich sie die Chance hochschätzen, immerhin einen Impuls zu setzen. In der Mehrheit der mündlichen Rückmeldungen drückte sich aus, dass die Ehrenamtlichen sich durch den Erfahrungsaustausch in ihrem bisherigen Handeln bestätigt und gestärkt fühlten. Das soll nicht als negatives Resultat gelten, lässt aber auch kein positives Resümee zu.

Da sich interkulturelle Kompetenz schwerlich messen oder in einer Prüfung abfragen lässt und ihr Erwerb von sehr vielen nicht zu vereinheitlichenden Faktoren abhängt (vgl. Hofmuth/Weber 2011), ist eine Aussage über die Wirkung einzelner interkultureller Fortbildungen nahezu unmöglich, insbesondere ohne eine umfangreiche Befragung der Teilnehmer/-innen und einen aussagekräftigen Prä-Post-Vergleich. Auf Basis der Beobachtungen lässt sich teilweise eine Veränderung hinsichtlich des Selbstreflexionsgrads der Teilnehmer/-innen feststellen, z.B. durch die Reformulierung mitgebrachter Statements oder bewussteren sprachlichen Ausdruck. Über einen längeren Zeitraum ließen

23 Anti-Bias-Werkstatt: „Was ist der Anti-Bias-Ansatz?“, online unter: http://www.anti-bias-werkstatt.de /?q=de/content/was-ist-der-anti-bias-ansatz [Stand: 11.03.2017]. 
sich Aspekte wie das regelmäßige Infragestellen der eigenen Perspektive und Haltung, die kritische Reflexion von Sprache, die Umsetzung erlernter kommunikativer Techniken etc. abfragen. Entsprechende Erhebungen werden laut den befragten Personen aus den hier betrachteten Einrichtungen nicht durchgeführt und sind sehr aufwändig. Eine aussagekräftige Evaluation läge, im Sinne einer Sicherung von Qualität und Nachhaltigkeit, in der Verantwortung der Träger/ Veranstalter bzw. des BAMF, das im Auftrag des Bundesministeriums des Inneren (BMI) und Bundesministeriums für Familie, Senioren, Frauen und Jugend (BMFSFJ) Projekte mit dem Schwerpunkt „Etablierung einer Willkommenskultur“ (vgl. Schünemann/Voigt/Huth 2016) fördert. Zu diesem Programm zählen auch Angebote zur Qualifizierung und Weiterbildung für Ehrenamtliche in der Integrationsarbeit, u.a. in Form von Multiplikatorenschulungen. ${ }^{24}$ Allerdings geben die von Seiten des BAMF aktuell verfügbaren Übersichten und Berichte keinen Aufschluss über die Wirksamkeit der durchführten Fortbildungen. ${ }^{25}$

Die Befragten haben das kompakte Format der Maßnahmen durchweg kritisch kommentiert - während sie die theoretische Grundlage bzw. inhaltliche Ausgestaltung ihrer Maßnahmen nicht in Zweifel zogen. Auf Nachfrage haben sie sich deutlich für Fortbildungen mit mehreren Modulen ausgesprochen, die aufeinander aufbauen. Eine solche Konzeption setzt voraus, dass eine Systematisierung und ggf. auch Bindung des (grundsätzlich von starker Fluktuation geprägten) Adressatenkreises erfolgt, würde aber eine Weiterentwicklung und Verbesserung der Angebote ermöglichen, die ohne diese Erfassung nicht fundiert und daher willkürlich wäre. Dazu muss angemerkt werden, dass sich viele Angebote zum Zeitpunkt dieser Studie in der Pilotierungsphase befanden, nicht zuletzt begründet durch noch zu wenig ausgeprägte Erfahrung der Anbieter und Trainerinnen mit dem Adressatenkreis. Im Zuge einer mittelbaren Flüchtlingshilfe durch Engagementförderung standen und stehen ausreichend finanzielle Mittel zur Verfügung, um neue Angebote zu schaffen. Doch leiden „Feuerwehrmaßnahmen“ bekanntlich an einem „Defizit an theoretischer Fundierung“ (vgl. Potthoff 2001). Hier besteht Entwicklungs- und Forschungsbedarf, um das Aktionsfeld der interkulturellen Qualifizierung von Freiwilligen zu verbessern und zu professionalisieren.

\section{Conclusio und Ausblick}

Die Trainerinnen der hier beobachteten Fortbildungen lieferten trotz ungünstiger struktureller Faktoren - starke Fluktuation der Teilnehmer/-innen, eng kalkulierter Zeitumfang u.a. - zahlreiche Beispiele guter Praxis, die sich für die Weiterentwicklung von Fortbildungen für diese spezielle Zielgruppe anbieten. Anhand der wenigen ganztägigen Veranstaltungen wurde deutlich, wie wertvoll dieser Raum ist, um diskursiv und kooperativ Begriffe zu erarbeiten, theoretische Modelle, z.B. durch Rollenspiele, explizit auf

24 Zusammenfassung in einer „Interaktive[n] Karte Multiplikatorenschulungen“, vgl. Bundesamt für Migration und Flüchtlinge (2016a).

25 Auf Nachfrage bestätigte eine Mitarbeiterin des Bundesamts für Migration und Flüchtlinge, dass eine entsprechende Statistik nicht vorliege, betonte aber, dass die Qualifizierung Ehrenamtlicher „,immer ein wichtiger Bestandteil“ der „Projekte mit dem Schwerpunkt der Etablierung einer Willkommenskultur" sei (zitiert aus einer Email von Theresa Haller, Referentin im Referat 321 - Integrationsprojekte [3. März 2017]). 
den Handlungskontext der Freiwilligen zu übertragen und in Gruppengesprächen zu konkreten Fällen Denk- und Reflexionsprozesse anzustoßen. In dem Spektrum der mitgebrachten Teilnehmerfragen, von denen viele unbeantwortet bleiben mussten, drückt sich die Breite von Lernbedürfnissen und die Unschärfe der Erwartungen an eine interkulturelle Fortbildung aus.

Aus dieser Beobachtung kann ein Argument für eine modulare Konzeption der Freiwilligenqualifizierung abgeleitet werden, die die Einbettung interkultureller Sensibilisierung in einen größeren thematischen Kontext vorsieht. Die positiven Erfahrungen von Trägern, die bereits mit solchen Konzepten arbeiten, unterstreichen dies. Auch für eine Verschränkung der interkulturellen Theorie mit anderen Ansätzen (die stärker auf Auseinandersetzung mit Privilegien der (weißen) Mehrheitsgesellschaft, Macht- und Dominanzkulturen, Mechanismen von Hierarchisierung usw. fokussieren) gab es, wie oben ausgeführt wurde, hilfreiche Exempel, die die Passung und Relevanz für den Adressatenkreis aufzeigen.

Wie schon erwähnt, liegen zu interkulturellen Sensibilisierungsmaßnahmen für Ehrenamtliche in der Flüchtlingsarbeit keine Daten und Auswertungen vor. Mit Perspektive auf eine langfristige, i.e. nachhaltige Unterstützung der freiwilligen Flüchtlingsarbeit durch Qualifizierungsmaßnahmen wäre eine wissenschaftlich fundierte Bestandsaufnahme erforderlich. Neben einer deutlichen Vergrößerung der Stichprobe sollte zunächst vor allem die Gruppe der Teilnehmer/-innen einbezogen und hinsichtlich ihres Erfahrungshorizonts, ihrer Erwartungshaltung und Zufriedenheit mit den bestehenden Angeboten sowie des subjektiv wahrgenommenen Lernerfolgs befragt werden.

\section{LITERATUR}

Anti-Bias-Werkstatt: Was ist der Anti-Bias-Ansatz?; online unter: http://www.anti-bias-werkstatt.de/?q=de /content/was-ist-der-anti-bias-ansatz [Stand: 02.03.2017].

Arndt, Susan (2005): Mythen des weißen Subjekts: Verleugnung und Hierarchisierung von Rassismus. In: Eggers, Maisha Maureen / Kilomba, Grada / Piesche, Peggy / Arndt, Susan (Hgg.): Mythen, Masken und Subjekte. Kritische Weißseinsforschung in Deutschland. Münster: Unrast Verlag.

Atali-Timmer, Fatoş / Mecheril, Paul (2015): Zur Notwendigkeit einer rassismuskritischen Sprache. Dossier Empowerment. In: Blog antifra* Debatte, Bildung, Vernetzung zu Migration und gegen Rassismus und Neonazismus; online unter: http://antifra.blog.rosalux.de/zur-notwendigkeit-einer-rassismuskritischen-sprache/ [Stand: 12.03.2017].

Bernhard, Nicole (2002): Interkulturelles Lernen und Auslandsaustausch - „Spielend“ zu interkultureller Kompetenz. In: Volkmann, Laurenz / Stierstorfer, Klaus / Gehring, Wolfgang (Hg.): Interkulturelle Kompetenz: Konzepte und Praxis des Unterrichts. Tübingen: Gunter Narr, 193-216.

Bolten, Jürgen (2007): Interkulturelle Kompetenz. Landeszentrale für Politische Bildung Thüringen: Erfurt.

Bosse, Elke (2009): Förderung von Linguistic Awareness of Cultures im Rahmen interkultureller Trainings. In: Bauer, Gerd Ulrich (Hg.): Standpunkte und Sichtwechsel. Festschrift für Bernd Müller-Jacquier zum 60. Geburtstag. München: Iudicium, 338-362.

Broden, Anne / Mecheril, Paul (2007): Migrationsgesellschaftliche Re-Präsentationen. Eine Einführung. In: Dies. (Hg.): Re-Präsentationen. Dynamiken der Migrationsgesellschaft. Düsseldorf: IDA-NRW, 7-28; online unter: www.ida-nrw.de/cms/upload/PDF/Re-Praesentationen.pdf [Stand: 15.03.2017].

Bruchhagen, Verena (2016): Diversity to go? Über die Unmöglichkeit, den Umgang mit Diversität im Vorbeigehen zu erledigen. In: Newsletter für Engagement und Partizipation in Deutschland, 10/2016; online unter: www.b-b-e.de/fileadmin/inhalte/aktuelles/2016/05/newsletter-10-bruchhagen.pdf [Stand 10.03.2017]. 
Bundesamt für Migration und Flüchtlinge (2016a): Interaktive Karte Multiplikatorenschulungen 2016; online unter: http://www.bamf.de/SharedDocs/Anlagen/DE/Downloads/Infothek/Integrationsprojekte/multipli katorenschulung-landkarte-2016.html?nn=2205880 [Stand: 12.03.2017].

Bundesamt für Migration und Flüchtlinge (2016b): Asylgeschäftsstatistik für den Monat April 2016; online unter: https://www.bamf.de/SharedDocs/Anlagen/DE/Downloads/Infothek/Statistik/Asyl/201604-statistik-anlage-asyl-geschaeftsbericht.pdf?_blob=publicationFile [Stand: 15.03.2017].

Bundeszentrale für politische Bildung (2016): Im Fokus: Flüchtlingsprojekte - Extra. Fördermittel für Flüchtlingsund Integrationsprojekte. Akquisos, Nr. 1/2016; online unter: https://www.bpb.de/partner/akquisos/222387 /foerdermittel [Stand: 10.03.2017].

Cicek, Arzu / Heinemann, Alisha / Mecheril, Paul (2014): Warum Rede, die direkt oder indirekt rassistische Unterscheidungen aufruft, verletzen kann. In: Hentges, Gudrun / Nottbohm, Kristina / Jansen, Mechthild M. / Adamou, Jamila (Hgg.): Sprache - Macht - Rassismus. Berlin: Metropol-Verlag, 309-327.

Daldrup, Till: Zäsur der deutschen Geschichte. In: ZEIT online, 9. März 2016; online unter: http://www .zeit.de/politik/ausland/2016-03/fluechtlingskrise-deutschland-bilanz-fluechtlingspolitik-zaesur [Stand: 18.03.2017].

Foitzik, Andreas (2013): Kompaktwissen Interkulturelle Kompetenz, 4/2013. Hg. von Aktion Jugendschutz Baden-Württemberg, Landesarbeitsstelle Baden-Württemberg. Stuttgart; online unter: www.ajs-bw.de /media/files/aktuell/2013/KW-Interkultur2013_L10.pdf [Stand: 15.01.2017].

Foroutan, Naika / Schäfer, Isabel (2009): Hybride Identitäten - muslimische Migrantinnen und Migranten in Deutschland und Europa. In: APuZ 5/2009 Lebenswelten von Migrantinnen und Migranten, 11-18.

Frisch, Max (1967): Überfremdung I. In: Ders.: Öffentlichkeit als Partner. Frankfurt a. M.: Suhrkamp, 100-104. Geißler, Rainer (2014): Migration und Integration. In: Bundeszentrale für politische Bildung (Hg.): Sozialer Wandel in Deutschland. Informationen zur politischen Bildung, Nr. 324/2014, 40-52; online unter: http://www.bpb.de/shop/zeitschriften/informationen-zur-politischen-bildung/197876/sozialer-wandel -in-deutschland [Stand: 02.03.2017].

Hall, Stuart (1992/1994): Die Frage der kulturellen Identität. In: Mehlem, Ulrich / Bohle, Dorothee / Gutsche, Joachim / Oberg, Matthias / Schrage, Dominik (Hgg.): Stuart Hall. Ausgewählte Schriften 2. Rassismus und kulturelle Identität. Hamburg: Argument Verlag, 180-222.

Hall, Edward T. / Hall, Mildred R. (1990): Understanding Cultural Differences. Germans, French and Americans. Yarmouth: Maine.

Herdel, Shantala (2007): Was ist Anti-Bias? In: Anti-Bias-Werkstatt (Hg.): CD-Methodenbox: Demokratie-Lernen und Anti-Bias-Arbeit, Berlin; online unter: http://www.anti-bias-werkstatt.de/?q=de/content/downloads [Stand: 11.03.2017]

Hofmann, Heidemarie / Mau-Endres, Birgit / Ufholz, Bernhard (2005): Schlüsselqualifikation Interkulturelle Kompetenz Arbeitsmaterialien für die Aus- und Weiterbildung. Leitfaden für die Bildungspraxis. Schriftenreihe der Beruflichen Fortbildungszentren der Bayerischen Wirtschaft (bfz) gGmbH, Band 8. Bielefeld: Bertelsmann.

Hofmuth, Matthias / Weber, Susanne (2011): Zur Messung interkultureller Kompetenz. In: Faßhauer, Uwe / Aff, Josef / Fürstenau, Bärbel / Wuttke, Eveline (Hgg.): Lehr-Lernforschung und Professionalisierung. Perspektiven der Berufsbildungsforschung. (Schriftenreihe der Sektion Berufs- und Wirtschaftspädagogik der Deutschen Gesellschaft für Erziehungswissenschaft (DGfE). Opladen: Budrich, 25-36.

Hofstede, Geert (2001): Culture's Consequences - Comparing Values, Behaviors, Institutions and Organizations Across Nations. Thousand Oaks: Sage Publications.

IJAB - Fachstelle für Internationale Jugendarbeit der Bundesrepublik Deutschland e.V:: Methodenbox Interkulturell; online unter: http://www.dija.de/toolbox-interkulturelles-lernen/methodenbox-interkulturell/ [Stand: 14.03.2017].

Karakayali, Serhat / Kleist, J. Olaf (2016): EFA-Studie 2: Strukturen und Motive der ehrenamtlichen Flüchtlingsarbeit in Deutschland, Forschungsbericht: Ergebnisse einer explorativen Umfrage vom November/Dezember 2015. Berlin: Berliner Institut für empirische Integrations- und Migrationsforschung (BIM), Humboldt-Universität zu Berlin; online unter: http://www.bim.hu-berlin.de/media/Studie_EFA2_BIM_11082016_V\%C3\%96 .pdf [Stand: 10.03.2017].

Kumbier, Dagmar / Schulz von Thun, Friedemann (Hgg.) (2006): Interkulturelle Kommunikation. Methoden, Modelle, Beispiele. Reinbek bei Hamburg: Rowohlt Verlag. 
Mecheril, Paul / do Mar Castro Varela, Maria / Inci, Dirim / Kalpaka, Annita / Melter, Claus (2010): Migrationspädagogik. Weinheim / Basel: Beltz.

Müller-Jacquier, Bernd (2000): Linguistic Awareness of Cultures. Grundlagen eines Trainingsmoduls. In: Bolten, Jürgen (Hg.): Studien zur internationalen Unternehmenskommunikation. Leipzig: Popp, 20-49.

Netzwerk Rassismuskritische Migrationspädagogik BW: Was ist rassismuskritische Migrationspädagogik?; online unter: http://www.rassismuskritik-bw.de/ueber-uns/positionierung/ [Stand: 11.03.2017].

Potthoff, Anke (2001): Analyse von Weiterbildungsangeboten zur Förderung interkultureller Kompetenz in der Verwaltung. In: Riehle, Eckart (Hg.): Interkulturelle Kompetenz in der Verwaltung? Kommunikationsprobleme zwischen Migranten und Behörden. Wiesbaden: VS Verlag für Sozialwissenschaften, 116-136.

Rosenberg, Katharina (2014): Interkulturelle Behördenkommunikation. Eine gesprächsanalytische Untersuchung $z u$ Verständigungsproblemen zwischen Migranten und Behördenmitarbeitern in Berlin und Buenos Aires. (Beihefte zur Zeitschrift für Romanische Philologie 380). Berlin / Boston: de Gruyter.

Ruch, Floyd L. / Zimbardo, Philip G. (1974): Lehrbuch der Psychologie. Eine Einführung für Studenten der Psychologie, Medizin und Pädagogik. Berlin / Heidelberg / New York: Springer Verlag.

Schünemann, Gabriele / Voigt, Birgit / Huth, Susanne (2016): Fachlich-wissenschaftliche Begleitung einer Projektreihe zur „Förderung des sozialen Zusammenhalts durch Etablierung einer Willkommenskultur". Abschlussbericht. Offenbach: Institut für berufliche Bildung, Arbeitsmarkt- und Sozialpolitik (INBAS GmbH).

Schründer-Lenzen, Agi (2009): Sprachlich-kulturelle Heterogenität als Unterrichtsbedingung. In: Fürstenau, Sara / Gomolla, Mechthild (Hgg.): Migration und schulischer Wandel: Unterricht. Wiesbaden: VS Verlag für Sozialwissenschaften, 121-138.

Simonson, Julia / Vogel, Claudia / Tesch-Römer, Clemens (Hgg.) (2016): Freiwilliges Engagement in Deutschland - Der Deutsche Freiwilligensurvey 2014. Hg. vom Bundesministerium für Familie, Senioren, Frauen und Jugend. Berlin: Springer-Verlag.

Springer Gabler Verlag (Hg.): Stichwort interkulturelles Training. In: Gabler Wirtschaftslexikon; online unter: http://wirtschaftslexikon.gabler.de/Archiv/4693/interkulturelles-training-v9.html [Stand: 10.03.2017].

Stiftung Bürgermut / Bundesarbeitsgemeinschaft der Freiwilligenagenturen e.V. (bagfa) (Hgg.) (2016): Refugees. Richtig gute Projekte, Tipps \& Tools, Berlin.

Thomas, Alexander (2003): Interkulturelle Kompetenz - Grundlagen, Probleme und Konzepte. In: Erwägen, Wissen, Ethik 14.1 (2003), 137-221.

Vertovec, Steven (2007): Super-diversity and its implications. In: Ethnic and Racial Studies 30, Nr. 6, November 2007, 1024-1054.

Vollmer, Michael (2015): Bestimmung von Fachkräfteengpässen und Fachkräftebedarfen in Deutschland, Studie der deutschen nationalen Kontaktstelle für das Europäische Migrationsnetzwerk (EMN). Working Paper 64 des Forschungszentrums des Bundesamtes. Nürnberg: Bundesamt für Migration und Flüchtlinge; online unter: http://www.bamf.de/SharedDocs/Anlagen/DE/Publikationen/EMN/Studien/wp64-emn -bestimmung-fachkrafteengpaesse-und-bedarfe.pdf?_blob=publicationFile [Stand: 05.03.2017]. 


\section{Anlagen (anonymisiert)}

Tab. 1: Interkulturelle Fortbildungsmaßnahmen für Ehrenamtliche in der Flüchtlingsarbeit (Auswahl)

\begin{tabular}{|c|c|}
\hline $\begin{array}{l}\text { Veranstaltungstitel/ } \\
\text { Datum, Uhrzeit/ Trainer/in(nen) }\end{array}$ & $\begin{array}{l}\text { Träger/ Anbieter/ } \\
\text { Quelle der Veröffentlichung }\end{array}$ \\
\hline $\begin{array}{l}\text { „Interkulturelle Kommunikation“ } \\
\text { 27.09.2016, 18-21 Uhr } \\
\text { Freiberufliche Interkulturelle Beraterin mit } \\
\text { den Schwerpunkten Beratung, interkulturelle } \\
\text { Schulungen, Organisation/ Betreuung }\end{array}$ & $\begin{array}{l}\text { Diakonie-Hilfswerk Hamburg, Migrations- } \\
\text { und Frauensozialarbeit, Projekt Flüchtlingslotsen } \\
\text { Quelle: http://aktivoli.kursportal.info/k4828541, } \\
\text { zuletzt: 18.03.2017 }\end{array}$ \\
\hline $\begin{array}{l}\text { „Einstiegsseminar Interkulturelle } \\
\text { Kommunikation“ } \\
\text { 21.10.2016, 16:00-19:00 Uhr } \\
\text { 22.10.2016, 10:00-13:00 Uhr } \\
\text { Leiterin von Seminaren zu interkultureller } \\
\text { Kommunikation, wissenschaftliche } \\
\text { Mitarbeiterin, Universität Bremen }\end{array}$ & $\begin{array}{l}\text { Evangelisches Bildungswerk Bremen } \\
\text { Quelle: Mailing durch das Evangelische } \\
\text { Bildungswerk Bremen }\end{array}$ \\
\hline $\begin{array}{l}\text { „Interkulturelle Sensibilisierung“ } \\
\text { 24.10.2016, 17:00-21:00 Uhr } \\
\text { keine Angabe zum/zur Trainer/in }\end{array}$ & $\begin{array}{l}\text { Diakonisches Werk Hamburg, Referat Migration, } \\
\text { Flucht und interkulturelle Arbeit } \\
\text { Quelle: http://aktivoli.kursportal.info/k4168921, } \\
\text { zuletzt: 18.03.2017 }\end{array}$ \\
\hline $\begin{array}{l}\text { „Interkulturelle Sensibilisierung“ } \\
\text { 08.09.2016, 18:00-21:00 Uhr } \\
\text { keine Angabe zum/zur Trainer/in }\end{array}$ & $\begin{array}{l}\text { DER PARITÄTISCHE Hamburg } \\
\text { Quelle: http://aktivoli.kursportal.info/k4168971, } \\
\text { zuletzt: } 10.09 .2016\end{array}$ \\
\hline $\begin{array}{l}\text { „Interkultureller Einstieg/ Sensibilisierung“ } \\
04.06 .2016,14: 00 \text { Uhr } \\
\text { erster Baustein in der Ausbildung zu } \\
\text { ehrenamtlichen Lernbegleitenden } \\
\text { keine Angabe zum/zur Trainer/in }\end{array}$ & $\begin{array}{l}\text { AFS Interkulturelle Begegnungen e.V. - } \\
\text { Normal ist die Vielfalt } \\
\text { Quelle: http://aktivoli.kursportal.info/k4459721, } \\
\text { zuletzt: 18.03.2017 }\end{array}$ \\
\hline $\begin{array}{l}\text { Basisseminar Flüchtlingsarbeit - Online-Seminar } \\
\text { ab 1. September } 2016 \\
\text { Seminar mit vier Modulen; u.a. } \\
\text { werden folgende Themen angekündigt: } \\
\text { interkulturelle Kompetenz; interreligiöse } \\
\text { Kompetenz, Anti-Bias-Methode (Ansatz der } \\
\text { antidiskriminierenden Bildungsarbeit) } \\
\text { Diplom-Kaufmann mit Schwerpunkt } \\
\text { Psychologie und Erwachsenenbildung, } \\
\text { IHK-zertifizierter Coach } \\
\text { Pflege- und Sozialcoach, Teamcoach, } \\
\text { Bildungsreferentin }\end{array}$ & $\begin{array}{l}\text { Caritasverband für die Stadt Köln e.V., } \\
\text { Caritas-Integrationsagentur } \\
\text { Quelle: http://www.fortbildung-caritasnet. } \\
\text { de/detail.php?kurs_nr=23716-309, zuletzt: } \\
\text { 18.03.2017 }\end{array}$ \\
\hline $\begin{array}{l}\text { Workshop „Interkulturelle Kompetenz“ } \\
\text { 11.07.2015, 9:30-15:00 Uhr } \\
\text { keine Angabe zum/zur Trainer/in }\end{array}$ & $\begin{array}{l}\text { Volkshochschule VHS Rüsselsheim } \\
\text { Quelle: https://www.facebook.com/events } \\
\text { /811511738945574/, zuletzt: } 18.03 .2017\end{array}$ \\
\hline $\begin{array}{l}\text { Integration durch interkulturelle Öffnung } \\
\text { 10.05.2016, 19:30-21:00 Uhr } \\
\text { Kulturen verstehen - Flüchtlingen helfen } \\
\text { 02.02.2016, 10:00-13:00 Uhr } \\
\text { Zertifizierte Trainerin für Interkulturelle } \\
\text { Kompetenz, CCS-Trainerin und Dozentin } \\
\text { für Deutsch als Fremdsprache }\end{array}$ & $\begin{array}{l}\text { Volkshochschule VHS Südost, Putzbrunn } \\
\text { Quelle: http://culturedecode.de/category } \\
\text { /aktuelle-trainings/, zuletzt: } 18.09 .2016\end{array}$ \\
\hline
\end{tabular}




\begin{tabular}{|c|c|}
\hline $\begin{array}{l}\text { „Interkulturelles Training“. Workshop } \\
\text { für Ehrenamtliche } \\
\text { 17./18.09.2016, 10:00-17:00 Uhr } \\
\text { Referent für Diversity Management im Referent } \\
\text { für Diversity Management im Aus- und } \\
\text { Fortbildungszentrum für den Bremischen } \\
\text { öffentlichen Dienst (AFZ) }\end{array}$ & $\begin{array}{l}\text { DRK Kreisverband Bremen e.V. } \\
\text { Quelle: Flyer des DRK KV Bremen e.V. }\end{array}$ \\
\hline $\begin{array}{l}\text { Workshop: Interkulturelle } \\
\text { Begegnungen - Alltagsrassismus } \\
\text { 18.11.2015, 14:15-17:45 Uhr } \\
\text { Teil des Zertifikatskurses „Studierende } \\
\text { unterstützen Flüchtlinge beim Spracherwerb“ } \\
\text { Trainerin, Hochschuldozentin }\end{array}$ & $\begin{array}{l}\text { Leuphana Universität Lüneburg } \\
\text { Quelle: http:// } \\
\text { www.leuphana.de/services/vorlesungsverzeichnis }\end{array}$ \\
\hline $\begin{array}{l}\text { Fortbildungen zu diversen Themen, von } \\
\text { unterschiedlicher Länge, darunter: } \\
\text { „Interkulturelle Verständigung } \\
\text { und interkulturelles Lernen" } \\
\text { „Umgang mit Normalität und Differenz" } \\
\text { "Kultursensibler Umgang mit Werten, } \\
\text { Erziehungszielen und -stilen“ } \\
\text { „Empathische Kommunikation } \\
\text { in interkulturellen } \\
\text { Begegnungen \& Konfliktsituationen" } \\
\text { keine Angabe zum/zur Trainer/in }\end{array}$ & $\begin{array}{l}\text { Hamburger Institut für interkulturelle Pädagogik } \\
\text { (HII) Hamburg } \\
\text { Quelle: http:// } \\
\text { www.hiip-hamburg.de/inhouse.html }\end{array}$ \\
\hline $\begin{array}{l}\text { „Interkulturelle Kommunikation für } \\
\text { Ehrenamtliche“ } \\
\text { 23.09.2016, 8:30-16:45 Uhr } \\
\text { Freiberufliche Dozentin und Beraterin für } \\
\text { Interkulturelle Kommunikation }\end{array}$ & $\begin{array}{l}\text { Landesbetrieb ZAF (Zentrum für Aus- und } \\
\text { Fortbildung)/ AMD, Forum Flüchtlingshilfe/ } \\
\text { BASFI (Behörde für Arbeit, Soziales, Familie und } \\
\text { Integration Hamburg) } \\
\text { Quelle: http://www.hamburg.de/forum } \\
\text {-fluechtlingshilfe/4919840, zuletzt: } 18.09 .2016\end{array}$ \\
\hline $\begin{array}{l}\text { „Workshop: BLICK IN DEN } \\
\text { SPIEGEL - BEGEGNUNG MIT RASSISMUS. } \\
\text { Schulung für Menschen in der Arbeit mit } \\
\text { Geflüchteten“ } \\
\text { 06.10.2016, 18:00-21:00 Uhr } \\
\text { Referent und Trainer zu Schwarzer Identität, } \\
\text { Critical Whiteness u.a. }\end{array}$ & $\begin{array}{l}\text { DER PARITÄTISCHE Hamburg } \\
\text { Quelle: http://www.paritaet-hamburg.de/ } \\
\text { fortbildungen/details/artikel/blick-in-den- } \\
\text { spiegel-begegnung-mit-rassismus.html, zuletzt: } \\
\text { 18.09.2016 }\end{array}$ \\
\hline $\begin{array}{l}\text { „Interkulturelles Training zur Selbstklärung } \\
\text { und als Argumentationshilfe gegen } \\
\text { Stammtischparolen und Vorurteile“ } \\
\text { November 2017, 18:00-21:00 Uhr } \\
\text { Baustein in „Brücken bauen - Ankommen } \\
\text { begleiten - Ein Kurs für Ehrenamtliche in der } \\
\text { Flüchtlingsarbeit“ } \\
\text { Fachstelle für Inklusion-Interkulturalität, } \\
\text { Jugendpfarramt in der Nordkirche }\end{array}$ & $\begin{array}{l}\text { Arbeitsstelle Ökumene - Menschenrechte - } \\
\text { Flucht - Friedensbildung der Nordkirche } \\
\text { Quelle: http://hamburg.kursportal.info/ } \\
\text { k4848731, zuletzt: } 18.03 .2017\end{array}$ \\
\hline
\end{tabular}


Tab. 2: Analyseraster für Interviews und Hospitationen

\begin{tabular}{|l|}
\hline Analysekriterien zum organisatorischen und situativen Rahmen der Fortbildungen \\
\hline Finanzierung der Maßnahmen (staatliche/ öffentliche oder kirchliche Gelder, Stiftungen) \\
Format und Umfang (Kompaktkurs, mehrteilig, modularer Aufbau, Stundenumfang) \\
Teilnehmer/-innen (Alter, Geschlecht, Beruf, sozialer und kultureller Hintergrund [POC \\
oder nicht], individuelle Lernvoraussetzungen, Engagementfeld, Eigenmotivation für das \\
Freiwilligenengagement, eigene Fragen und Themen) \\
Trainer/-innen (Alter, Geschlecht, Qualifikation/ Ausbildung, kultureller Hintergrund [POC oder \\
nicht], Erfahrung mit Freiwilligenqualifizierung, Erfahrung mit Freiwilligen/ Professionellen \\
in der Flüchtlingsarbeit) \\
Nachfrage und Reichweite des Angebots \\
Qualitätssicherung (Evaluationsverfahren/ Akkreditierung, Qualifikation/ Professionalität \\
der Trainer/-innen, Zertifikat für die Teilnehmer/-innen) \\
\hline Analysekriterien zu Inhalten, Methoden und Zielen der Vermittlung \\
\hline Inhalte und Themen (Kulturbegriff, Stereotype und Vorurteile, Themen mit Bezug \\
zur Freiwilligenarbeit für Geflüchtete) \\
Vermittlungsziele (Vermittlung von Wissen: Eigen- und Fremdkulturwissen, Wissen über \\
Kommunikationskulturen; Vermittlung von Fertigkeiten: interkulturelle Kompetenz, \\
Ambiguitätstoleranz, Fähigkeit zum Perspektivwechsel, Kulturverstehen; Entwicklung von \\
Haltungen/Einstellungen) \\
Theoretische Basis (Terminologie, Kulturerklärungsansätze und -modelle, \\
Interkulturalitätskonzepte) \\
Methode, Didaktik, pädagogischer Ansatz (Vortrag, Gruppenarbeit, Rollenspiele, Simulationen, \\
Kollegiale Beratung, Reflexionsformate, partizipative, interaktive, spielerische Methoden u.a.) \\
\hline
\end{tabular}


Tab. 3: Fragensets für Leitfadeninterviews

\section{Fragenset für Interviews mit Vertreter/-innen von Trägereinrichtungen}

Welche Angebote zur interkulturellen Sensibilisierung machen Sie für Freiwillige in der ehrenamtlichen Flüchtlingsarbeit?

Welche Ziele verfolgen Sie mit Ihren Angeboten?

Welche Inhalte und Formate sehen Sie vor?

Wie setzt sich Ihre Zielgruppe zusammen?

Welche Wissensdefizite bzw. Schulungsbedarfe nehmen Sie bei der Zielgruppe wahr?

Wie entwickelt sich die Nachfrage? Konstant oder schwankend?

Führen Sie Evaluationen durch? Wie fallen dieses aus?

Erhalten die Teilnehmer ein Zertifikat?

Können Sie den Lernerfolg der Teilnehmer/-innen beurteilen?

Wie finanzieren Sie die Angebote?

Wie lange können Sie diese perspektivisch aufrechterhalten?

\section{Fragenset für Interviews mit Trainer/-innen}

Wie setzt sich Ihre Zielgruppe zusammen?

Welche Wissensdefizite bzw. Schulungsbedarfe nehmen Sie bei der Zielgruppe wahr?

Nehmen Sie bei den Teilnehmern wiederkehrende Fragen wahr?

Wie reflektiert, offen bzw. selbstkritisch erleben Sie die Teilnehmer?

Sind Sie mit den möglichen Formaten zufrieden? Beurteilen Sie diese

als adäquat?

Welche Inhalte und Themen behandeln Sie in Ihren Schulungen?

Welche Modelle und Methoden verwenden Sie? Welche eignen sich besonders?

In welchem Verhältnis stehen Input und erfahrungs-/ interaktionsorientierte Anteile?

Holen Sie während/ nach der Schulung Feedback ein? Wie fällt dieses aus?

Können Sie den Lernerfolg der Teilnehmer/-innen beurteilen? 
Tab. 4: Interviews mit Weiter- und Fortbildungsträgern und Trainer/innen (2015/2016)

Umfang der vorliegenden Daten: Protokolle aus 11 Interviews von jeweils 30 bis 90 Minuten Dauer; Auswahlkriterien: Finanzierung aus öffentlichen/ staatlichen oder kirchlichen Geldern, exklusive Konzeption für die Zielgruppe ehrenamtlich in der Flüchtlingsarbeit Engagierter, Durchführung durch professionelle Trainer/-innen.

\begin{tabular}{|c|c|}
\hline Datum/ Uhrzeit & $\begin{array}{l}\text { Interviewpartner/in, Funktion/ Position bzw. Titel/ } \\
\text { Qualifikation, Einrichtung/ Auftraggeber/in. } \\
\text { Form des Interviews, Ort/Ort. }\end{array}$ \\
\hline $\begin{array}{l}12.04 .2016 \\
10: 00-11: 30 \mathrm{Uhr}\end{array}$ & $\begin{array}{l}\text { Zertifizierte Trainerin, selbständig. Präsenzinterview, Hamburg/ } \\
\text { Hamburg. }\end{array}$ \\
\hline $\begin{array}{l}11.08 .2016 \\
11: 30-12: 30 \mathrm{Uhr}\end{array}$ & $\begin{array}{l}\text { Leiterin der Caritas-Integrationsagentur im Caritas-Zentrum Kalk, } \\
\text { Caritasverband für die Stadt Köln e.V. Telefoninterview, } \\
\text { Köln/Hamburg. }\end{array}$ \\
\hline $\begin{array}{l}\text { 11.08.2016 } \\
17: 00-17: 45 \mathrm{Uhr}\end{array}$ & $\begin{array}{l}\text { Projektleitung Qualifizierung und Beratung Freiwilliger in der } \\
\text { Arbeit mit Geflüchteten, Der PARITÄTISCHE Wohlfahrtsverband } \\
\text { Hamburg e.V. Telefoninterview, Hamburg/Hamburg. }\end{array}$ \\
\hline $\begin{array}{l}\text { 11.08.2016 } \\
\text { 09:00-10:30 Uhr }\end{array}$ & $\begin{array}{l}\text { Trainerin für Interkulturelle Kommunikation, Leiterin } \\
\text { Integrationskurse, VHS Putzbrunn, Landkreis München. } \\
\text { Telefoninterview, München/Hamburg. }\end{array}$ \\
\hline $\begin{array}{l}24.08 .2016 \\
14: 30-15: 20 \mathrm{Uhr}\end{array}$ & $\begin{array}{l}\text { Professionelle Trainerin, interCultur gGmbH. Telefoninterview, } \\
\text { Hamburg/Hamburg. }\end{array}$ \\
\hline $\begin{array}{l}\text { 24.08.2016 } \\
\text { 10:00-11:00 Uhr }\end{array}$ & $\begin{array}{l}\text { Interkulturelle Trainerin, Freiberufliche Dozentin und Beraterin für } \\
\text { interkulturelle Kommunikation, Mediatorin, Systemischer Coach. } \\
\text { Telefoninterview, Hamburg/Hamburg. }\end{array}$ \\
\hline $\begin{array}{l}\text { 24.08.2016 } \\
\text { 10:00-11:00 Uhr }\end{array}$ & $\begin{array}{l}\text { Zertifizierte Trainerin, Systemische Organisationsberaterin, } \\
\text { selbständig. Telefoninterview, Hamburg/Hamburg. }\end{array}$ \\
\hline $\begin{array}{l}25.08 .2016 \\
11: 00-12: 00 \mathrm{Uhr}\end{array}$ & $\begin{array}{l}\text { Pädagogische Mitarbeiterin, zuständig für Flüchtlingskurse, } \\
\text { Katholisches Bildungswerk Köln. Telefoninterview, Köln/Hamburg. }\end{array}$ \\
\hline $\begin{array}{l}\text { 31.08.2016 } \\
13: 00-13: 45 \mathrm{Uhr}\end{array}$ & $\begin{array}{l}\text { Ansprechpartner für Informatik und Medien, Oldenburger } \\
\text { Fortbildungszentrum (OFZ), Carl von Ossietzky Universität } \\
\text { Oldenburg }\end{array}$ \\
\hline $\begin{array}{l}\text { 28.09.2016 } \\
\text { 10:00-11:00 Uhr }\end{array}$ & $\begin{array}{l}\text { Interkulturelle Trainerin, Diakonisches Werk Hamburg. } \\
\text { Telefoninterview, Hamburg/Hamburg }\end{array}$ \\
\hline $\begin{array}{l}\text { 03.10.2016 } \\
15: 30-17: 00 \mathrm{Uhr}\end{array}$ & $\begin{array}{l}\text { Zertifizierte Trainerin, selbständig. Telefoninterview, Hamburg/ } \\
\text { Hamburg. }\end{array}$ \\
\hline $\begin{array}{l}16.08 .2018 \\
12: 30-13: 30 \mathrm{Uhr}\end{array}$ & $\begin{array}{l}\text { Professor an der Evangelischen Hochschule für Soziale Arbeit } \\
\text { \& Diakonie in Hamburg sowie wissenschaftlicher Leiter des } \\
\text { Hamburger Instituts für Interkulturelle Pädagogik (HIIP). } \\
\text { Telefoninterview, Hamburg/Hamburg. }\end{array}$ \\
\hline
\end{tabular}


Tab. 5: Hospitationen (2015 bis 2017)

Umfang der vorliegenden Daten: Protokolle von 5 Hospitationen in Fortbildungen von jeweils drei Stunden Dauer, alle in Hamburg; Auswahlkriterien: Finanzierung aus öffentlichen, staatlichen oder kirchlichen Geldern, exklusive Konzeption für die Zielgruppe ehrenamtlich in der Flüchtlingsarbeit Engagierter, Durchführung durch professionelle Trainer/-innen.

\begin{tabular}{|c|c|}
\hline Datum/ Uhrzeit/ Dauer & Titel der Fortbildung. Träger/ Veranstalter. Trainerin(nen) \\
\hline $\begin{array}{l}\text { 20.07.2015 } \\
\text { 18:00-21:00 Uhr } \\
\text { 3 Stunden }\end{array}$ & $\begin{array}{l}\text { Interkulturelle Sensibilisierung. Diakonisches Werk } \\
\text { Hamburg - Referat Migration, Flucht und interkulturelle Arbeit. }\end{array}$ \\
\hline $\begin{array}{l}\text { 30.11.2015 } \\
14: 15-17: 45 \text { Uhr } \\
\text { 3 Stunden }\end{array}$ & $\begin{array}{l}\text { Umgang mit sprachlicher und kultureller Verschiedenheit } \\
\text { in der ehrenamtlichen Flüchtlingsarbeit. Universität Hamburg, } \\
\text { Seminar Refugees welcome - aber wie? Qualifizierung für } \\
\text { Studierende, die sich freiwillig für Geflüchtete engagieren. }\end{array}$ \\
\hline $\begin{array}{l}\text { 23.05.2016 14:15-17:45 Uhr } \\
3 \text { Stunden }\end{array}$ & $\begin{array}{l}\text { Interkulturelle Sensibilisierung für die Arbeit mit Geflüchteten. } \\
\text { Universität Hamburg, Seminar Refugees welcome - aber wie? } \\
\text { Qualifizierung für Studierende, die sich freiwillig für Geflüchtete } \\
\text { engagieren. }\end{array}$ \\
\hline $\begin{array}{l}\text { 26.05.2016 } \\
\text { 18:00-21:00 Uhr } \\
\text { 3 Stunden }\end{array}$ & Interkulturelle Sensibilisierung. Der PARITÄTISCHE Hamburg. \\
\hline $\begin{array}{l}\text { 27.09.2016 } \\
\text { 18:00-21:00 Uhr } \\
\text { 3 Stunden }\end{array}$ & $\begin{array}{l}\text { Interkulturelle Kommunikation. Projekt Flüchtlingslotsen des } \\
\text { Diakonischen Werks Hamburg. Fortbildung für ehrenamtliche } \\
\text { Begleiter/-innen von geflüchteten und migrierten Menschen } \\
\text { in Hamburg. }\end{array}$ \\
\hline $\begin{array}{l}25.02 .2017 \\
\text { 8:30-16:45 Uhr } \\
\text { 8 Stunden }\end{array}$ & $\begin{array}{l}\text { Interkulturelle Kommunikation für Ehrenamtliche. Landesbetrieb } \\
\text { ZAF/AMD. Zentrum für Aus- und Fortbildung. Forum } \\
\text { Flüchtlingshilfe. Fortbildungsveranstaltungen für Flüchtlingshelfer/ } \\
\text {-innen. }\end{array}$ \\
\hline
\end{tabular}


Tab. 6: Themen und Fragen, die die Ehrenamtlichen mitbringen (Auswahl)

\begin{tabular}{|c|c|}
\hline Themen & Zitate von Teilnehmer/-innen \\
\hline Begrüßung & $\begin{array}{l}\text { Ich weiß, dass die mir nicht die Hand geben, weil sie religiös sind. Aber } \\
\text { mich verletzt das trotzdem. Das ist hier in Deutschland einfach nicht } \\
\text { normal. }\end{array}$ \\
\hline Pünktlichkeit & $\begin{array}{l}\text { Wie erkläre ich denen, dass man in Deutschland pünktlich sein muss, } \\
\text { wenn man einen Termin hat? }\end{array}$ \\
\hline Zuverlässigkeit & $\begin{array}{l}\text { Es ist schon so oft passiert, dass wir einen Termin hatten und ich dann } \\
\text { alleine am Treffpunkt stand. Ich muss meine Zeit aber doch auch } \\
\text { einteilen. Wenn ich mich nicht auf eine Zusage verlassen kann, ist das } \\
\text { schwierig. }\end{array}$ \\
\hline Dankbarkeit & $\begin{array}{l}\text { Manchmal finde ich die auch sehr undankbar. Man bemüht sich so sie } \\
\text { zu unterstützen, aber nicht immer bekommt man dafür Wertschätzung }\end{array}$ \\
\hline Privater Wohnraum & $\begin{array}{l}\text { Muss ich denn, wenn ich die im Container besuche, auch draußen } \\
\text { die Schuhe ausziehen? }\end{array}$ \\
\hline Bild/Rolle der Frau & $\begin{array}{l}\text { Gerade die jungen arabischen Männer behandeln mich nicht } \\
\text { respektvoll. Ich finde, das müssen die hier lernen, dass eine Frau } \\
\text { genauso viel wert ist wie ein Mann. } \\
\text { An den freiwilligen Sprachkursen nehmen so wenige Frauen teil. } \\
\text { Wie kann man daran etwas ändern? }\end{array}$ \\
\hline $\begin{array}{l}\text { Machstrukturen } \\
\text { aufbrechen }\end{array}$ & $\begin{array}{l}\text { Dieses Helfen kommt natürlich immer von oben. Das ist für viele auch } \\
\text { nicht so einfach. Eigentlich müssen wir versuchen einander } \\
\text { auf Augenhöhe zu begegnen. }\end{array}$ \\
\hline $\begin{array}{l}\text { Abgrenzung, Neinsagen, } \\
\text { Verarbeitung }\end{array}$ & $\begin{array}{l}\text { Wie kann ich höflich erklären, dass ich nicht schon wieder zum } \\
\text { Abendessen bleiben will, ohne die Flüchtlingsfamilie zu verletzen? } \\
\text { Die Menschen haben so viel Schlimmes erlebt. Das erzählen sie dann } \\
\text { natürlich, sie müssen doch drüber reden. Wie kann ich selbst mit den } \\
\text { vielen Bildern im Kopf umgehen? Die belasten mich sehr. }\end{array}$ \\
\hline Tabus & $\begin{array}{l}\text { Soll man mit Flüchtlingen über ihre Flucht sprechen? Oder ist das } \\
\text { nicht so gut? }\end{array}$ \\
\hline Trauma & Wie soll man mit traumatisierten Geflüchteten umgehen? \\
\hline Religion & $\begin{array}{l}\text { (Wie) Soll man über Religion sprechen? Birgt das nicht ganz viel } \\
\text { Konfliktpotenzial? } \\
\text { Die tun ja so liberal, aber im Grunde, glaub ich, sind sie schon sehr } \\
\text { religiös. Das sieht man ja auch im Ramadan. }\end{array}$ \\
\hline Rassismus & $\begin{array}{l}\text { Wie soll ich mit rassistischen Haltungen umgehen, die ich bei den } \\
\text { Geflüchteten untereinander wahrnehme? }\end{array}$ \\
\hline Sexualität & $\begin{array}{l}\text { Wie soll man mit Komplimenten und Flirts von, südländischen } \\
\text { Männern` umgehen? }\end{array}$ \\
\hline
\end{tabular}

\section{Cornelia Springer}

Universität Hamburg, Fakultät für Geisteswissenschaften

Projektleitung „Engagementförderung durch universitäre Lehre“ (gefördert vom Bundesministerium für Bildung und Forschung im Rahmen des Qualitätspakts Lehre unter dem Förderkennzeichen 01PL17033)

cornelia.springer@uni-hamburg.de 